\title{
Constrained design of sheet forming processes
}

\author{
Evripides G. Loukaides ${ }^{1, a}$ and Julian M. Allwood ${ }^{1}$ \\ ${ }^{1}$ Department of Engineering, University of Cambridge, CB2 1PZ, United Kingdom
}

\begin{abstract}
New forming machines are typically incremental improvements on existing designs. Frequently tedious simulation and testing are required for insights on those possible improvements. A method for inventing features of flexible sheet forming processes is explored here. The inverse problem is solved by starting with a desired geometry, and "un-forming" the part while applying a selection of kinematic constraints and minimizing the plastic work on the workpiece. The resulting deformation reveals the required forces on the part and hence can inform machine design.
\end{abstract}

\section{Introduction}

Many modern forming technologies are inflexible which leads to significant material waste [1]. Reviews of existing flexible processes abound, especially with a focus in past decades on infusing modern technologies with the flexibility of traditional craftsmen [2-6]. However, progress remains slow and inconsistent. Sheet metal forming could greatly benefit from automated means of inventing new forming processes that can adhere to pre-determined constraints of deformation.

Such a method, based on the "un-forming" of target parts while minimizing the plastic work done on the workpiece was suggested in [7]. Here, the results of one iteration of the method are detailed and critiqued, with the intention of converging to a more robust method and eventually to a practically achievable process.

The idea of un-forming the part to reveal the underlying mechanics was inspired by similar work originating in the garment industry [8-9]. A simplified mass-and-spring model is used to describe a 3D surface and its elastic deformation, thus allowing evaluation of regions of high strain and subsequently of optimal cutting patterns. But the problem of parametrizing a 3D surface to a $2 \mathrm{D}$ plane is also an important field in applied mathematics, originating in cartography, but with modern implications for computer graphics. Again, many methods supply some simple material properties to the surface to define an objective function but do not strictly correspond to physical objects [10-12].

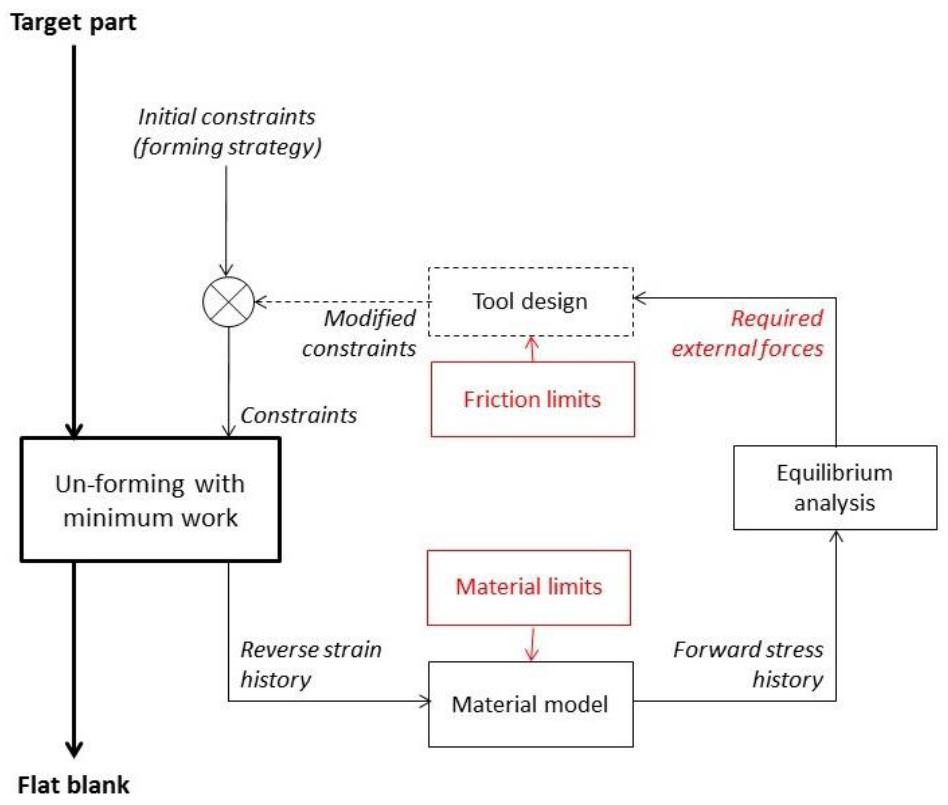

Figure 1. Block diagram of the "un-forming" method, adapted from [7]. The red text indicates the focus of this paper.

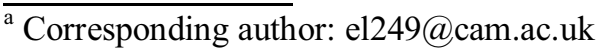


Inverse Finite Element Methods have been used in metal forming, but mostly for blank design. Minimization of plastic deformation is used to that end in [13]; a work guided by Hencky's deformation theory and Hill's anisotropic yield criterion. With the same objective Cai et al [14] used deformation non-uniformity as an objective function. The objective function in [7] draws from the work by Chung and Richmond $[15,16]$, which states that the optimal forming processes - under certain assumptions - are characterised by minimal deformation energy across the workpiece.

The assumptions and the implementation of the method in [7] are re-stated here for completeness, but the focus here is on the evaluation of the initial results and the potential for improvements.

\section{Method for process design by un- forming}

This paper examines the method shown in Figure 1 and originally suggested in [7]. An optimization strategy for the plastic work done is employed to "un-form" a selected part towards a flat blank. A combination of constraints are chosen to describe desired deformation characteristics and to direct the movement of the material towards a single plane.

The method in the diagram is iterative: After the unforming completes, the un-forming is reversed to reveal what the corresponding forming process would look like. Then, the feasibility and requirements for such a process are evaluated and the constraints are adjusted to attempt a subsequent "un-forming." This is repeated until both the desired constraints are satisfied and a feasible process is described.

The algorithm is described in great detail in [7], but a brief overview of the formulation is presented here for clarity and completeness. The focus of this paper is on the evaluation of one such un-forming, and specifically the metrics shown in red on Figure 1. They will be described and calculated in later Sections.

\subsection{Formulation for optimally un-forming subject to constraints}

Although the method can be used on any initial geometry for a surface (i.e. for any formed part), the work here uses results from [7] and maintains the selection of the square cup shape. The particular geometry contains developable and non-developable regions and a flange at the perimeter, thus making it both a common and challenging part in the sheet forming industry.

The square cup shape along with the various dimensions is shown in Figure 2. In the same Figure, a triangular discretization is shown and is used throughout [7] and this work.

The material law does not allow for an elastic region and takes the form:

$$
\sigma_{f}=\sigma_{0}\left(0.05+\bar{\epsilon}_{E}\right)^{n}
$$

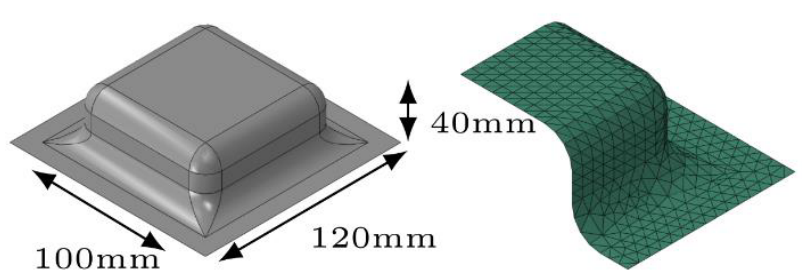

Figure 2. The square cup geometry used for all the simulations - modified from [7].

where $\bar{\epsilon}_{E}$ is the equivalent accumulated plastic strain. The parameters were modified from data in [17] and are based on the behaviour of aluminium 1100-O. These correspond to $\sigma_{0}=172 \mathrm{MPa}$ and $n=0.25$. Only an isotropic material is considered.

Since the modelling beings with the formed part, a distribution of plastic strain has to be assumed. If correct, the ensuing deformation should result in a flat blank with no plastic strain. There is no direct way to obtain these strains and in [7] an iterative approach is used to estimate a reasonable starting strain field that results in a mean blank strain field close to zero. The first guess in this process is a uniform field of $\bar{\epsilon}_{E}=\bar{\epsilon}_{E 0}^{(1)}=0.5$. The algorithm then takes the final field, $\bar{\epsilon}_{E f}$, from each iteration and subtracts is from the last starting field:

$$
\bar{\epsilon}_{E 0}^{(r)}=\bar{\epsilon}_{E 0}^{(r-1)}-\bar{\epsilon}_{E f}^{(r-1)}
$$

with $r$ being the current step in the iteration. The material law was adapted to prevent inadmissible negative strains from halting the calculation:

$$
\sigma_{f}=\sigma_{0}\left(0.05+\max \left\{\bar{\epsilon}_{E}, 0\right\}\right)^{n}
$$

The calculation of the plastic work for each element is given at each time step as:

$$
d W=\sigma_{f} d \bar{\epsilon}_{E} d V
$$

where $d V$ is the element volume and $d \bar{\epsilon}_{E}$ is the equivalent plastic strain decrement. To calculate the equivalent strain for each element, the concept of the constant strain triangle is adopted. The strains for each edge of the triangular element can be calculated from the Cartesian coordinates $\boldsymbol{x}$, as the vector $\boldsymbol{\epsilon}=\left[\boldsymbol{\epsilon}_{1}, \boldsymbol{\epsilon}_{2}, \boldsymbol{\epsilon}_{3}\right]$, such that

$$
\epsilon_{i}=\ln \left(\left\|x_{j}^{t_{n}}-x_{k}^{t_{n}}\right\|_{2} /\left\|x_{j}^{t_{n-1}}-x_{k}^{t_{n-1}}\right\|_{2}\right)
$$

with $x_{j}$ and $x_{k}$ being node coordinate on edge $i$ of the element, and the superscripts indicate the time step. The stretching effects are assumed to dominate the plastic work needed and no bending component is considered for the calculation.

The corresponding Cartesian strain field for the element can be calculated via the "rosette" transformation, $T_{e}$ : 


$$
d e=\left[\begin{array}{c}
d e_{x x} \\
d e_{y y} \\
2 d e_{x y}
\end{array}\right]=T_{e} \epsilon
$$

where

$$
T_{e}=\left[\begin{array}{ccc}
c_{1}^{2} & \mathrm{~s}_{1}^{2} & \mathrm{~s}_{1} \mathrm{c}_{1} \\
c_{2}^{2} & s_{2}^{2} & \mathrm{~s}_{2} \mathrm{c}_{2} \\
c_{3}^{2} & s_{3}^{2} & \mathrm{~s}_{3} \mathrm{c}_{3}
\end{array}\right]
$$

and the subscripted $c$ and $s$ correspond to the cosines and sines of the element edges to a local Cartesian system of axes.

Once the Cartesian components of plastic strain are obtained, an equivalent plastic strain for each element is calculated as:

$$
\begin{aligned}
& d \bar{\epsilon}=d \epsilon_{e q}^{p} \\
& =\sqrt{\frac{2}{3}\left[\left(d e_{x x}^{p}\right)^{2}+\left(d e_{y y}^{p}\right)^{2}+2\left(d e_{x y}^{p}\right)^{2}\right]}
\end{aligned}
$$

Then, returning to Equation (4), the plastic work on the element can be calculated and summed to produce the total plastic strain across the workpiece. The minimization problem is defined as

$$
\arg \min _{x \in \Omega} f=\sum_{i=1}^{n} d W_{i}
$$

subject to

$$
\begin{aligned}
& A_{i}^{t_{k}}=A_{i}^{t_{0}}, \forall i \in \Omega \\
& x_{p}^{t_{k}}=\mathrm{C}, \forall p \in \omega, \omega \in \Omega
\end{aligned}
$$

The constraint in Equation (10) represents a constant area requirement for each element throughout the simulation, which in turn means the deformation is in pure shear and there is no change in thickness under the constant volume assumption from metal plasticity theory. Certain known processes, such as spinning [18], approach this behaviour. At the same time, Forming Limit Diagrams show no established failure boundary in the pure shear direction [19], thus this mode allows for maximum local deformation.

The second constraint was pasted here in a general form and represents the strategy that is followed in gradually un-forming the workpiece. This usually involved constraining a set of nodes, forming the node set $\omega$, to a specific trajectory as to facilitate the un-forming, while allowing the rest of the nodes in the part, to move, as to minimize the objective function. Symmetry constraints are also applied to the xz and yz planes.

The solution to the optimization problem is attempted with the fmincon function in MATLAB which allows for a non-linear objective function as well as non-linear constraints. Within that, the 'interior-point' algorithm is used. The options give to fmincon are displayed in Table 1 .

In practise, the optimization at each step is first attempted for a zero objective function as to obtain a feasible starting point for a subsequent attempt with the objective function in Equation (9).
Table 1. fmincon options for optimization

\begin{tabular}{|l|c|}
\hline MaxFunEvals & $1 \mathrm{e} 8$ \\
\hline TolCon & $1 \mathrm{e}-3$ \\
\hline TolFun & $2 \mathrm{e}-1$ \\
\hline TolX & $1 \mathrm{e}-6$ \\
\hline MaxIter & $1 \mathrm{e} 5$ \\
\hline MaxFunEvals & $1 \mathrm{e} 8$ \\
\hline TolCon & $1 \mathrm{e}-3$ \\
\hline
\end{tabular}

The result of the optimization gives the updated geometry at each step and the equivalent decrement in equivalent plastic strain, which in turn allows for the calculation of the current plastic strain field:

$$
\bar{\epsilon}^{\mathrm{t}_{\mathrm{k}}}=\bar{\epsilon}^{\mathrm{t}_{\mathrm{k}-1}}-d \bar{\epsilon}^{\mathrm{t}_{\mathrm{k}-1}}
$$

\subsection{Evaluation and refinement of constraints}

After following the procedure in the previous Section, a complete history of displacement during un-forming is obtained. This can now be reversed and be considered the history of forming of the part from a flat blank. For each forward step in the forming, an incremental strain field can be calculated by comparing successive node coordinates, as defined in Equations (5-8).

A stress field at each step can then be produced at each step. The flow rule is defined as

$$
d \epsilon_{i j}^{p}=\lambda \sigma_{i j}^{\prime}
$$

where $\sigma_{i j}^{\prime}$ represents the deviatoric stresses and $\lambda$ is given by

$$
\lambda=\frac{3 d \epsilon_{e q}^{p}}{2 \sigma_{f}}=\frac{3 d \epsilon_{e q}^{p}}{2 \sigma_{0} \bar{\epsilon}^{n}}
$$

Thus, the deviatoric stresses are given as

$$
\sigma_{i j}^{\prime}=\frac{2 d \epsilon_{i j}^{p} \sigma_{0} \bar{\epsilon}^{n}}{3 d \epsilon_{e q}^{p}}
$$

However, to obtain a unique, complete stress field on the workpiece, an elastoplastic material model has to be considered. The calculation is straightforward in a displacement-controlled Finite Element model of the forming process. This not only produces the stress state of each element, but the required external nodal forces to achieve the suggested deformation.

The FE model was constructed in Abaqus as a single Dynamic Explicit step, with tabulated amplitude providing the location of each node at each time step. S3R elements were used. The material is also provided in tabulated form and follows the properties given for $\mathrm{Al}$ 1100 as above, but with a Young's Modulus of 71GPa 
and a Poisson's ratio of 0.33 . The thickness of the sheet was set at $1 \mathrm{~mm}$.

\section{Un-forming strategies}

In order to un-form the part the model must be given some perturbation that encourages the motion of the nodes towards a common plane. Three strategies were tried in [7] and are all shown in Figure 3: (a) The flattening of nodes is attempted locally, starting from regions of high negative Gaussian curvature and proceeding accordingly; (b) Un-form the part by moving the peripheral nodes radially outwards from the centre.; (c) Similar to (b) but with a variation of the out-wards trajectory. Here, only results from (b) are considered for further analysis, hence the strategy is presented in greater detail.

\subsection{Un-forming via radial boundary pull}

Deep drawing is often used to form the square cup and some inspiration can be found in examining relevant results. The boundaries of the blank move towards the deeper locations on the die. Thus, in un-forming the same process would require the boundaries to move away from the centre of the workpiece.

One strategy in [7] is based on pulling the nodes on the periphery on a radial trajectory away from the centre of the cup. First, the radial path connecting each peripheral node to the centre is found and its length, $u$, is recorded. The end point for the trajectory is placed on the same xy-plane as the centre of the workpiece, such that it is radially at a distance $u$ from the centre and on the same radial plane as the corresponding peripheral node. A linear path is defined between the two and the trajectory of the peripheral node is given at constant increments along this path. The same is done for all peripheral nodes and their motions are synchronised. The radial component of the peripheral motion ensures that gradually the depth of the part is reduced and it deforms towards a blank.

\subsection{Results for "radial pulling" strategy}

While the work in [7] demonstrated that the un-forming can be achieved with the method in Figure 1, the results of only the first iteration where shown. Those results are shown again here in Figure 4 for completeness. The diagrams (a-d) show steps 1, 29, 49 and 65 of the simulation (out of 65), starting in effect from the desired part geometry and ending with the blank. The remainder of this paper deals with the question "Which metrics are best to evaluate these results and inform the choice of constraints for the next iteration?"

\section{Metrics for evaluation of optimization and current constraints}

In Figure 1, the path to analysing the deformation history is shown on the right. In red lettering, specific metrics are noted that can inform us on the feasibility of the method and the practical implications of the results. The 'interior point' algorithm used for the optimization will not be evaluated here in depth, but the solver does return a feasible solution at each step, with the tolerances provided in Table 1, and a positive 'exitflag', indicating convergence or the step size reaching the 'TolX' tolerance.

The strains can be obtained directly, since the method produces a full deformation history. Then, the material model and equilibrium are employed to produce the stress field on the workpiece and the required external forces. In practise, an FEM model described in Section 2.2 is used to produce all output. Three potential ways of evaluating

(a)
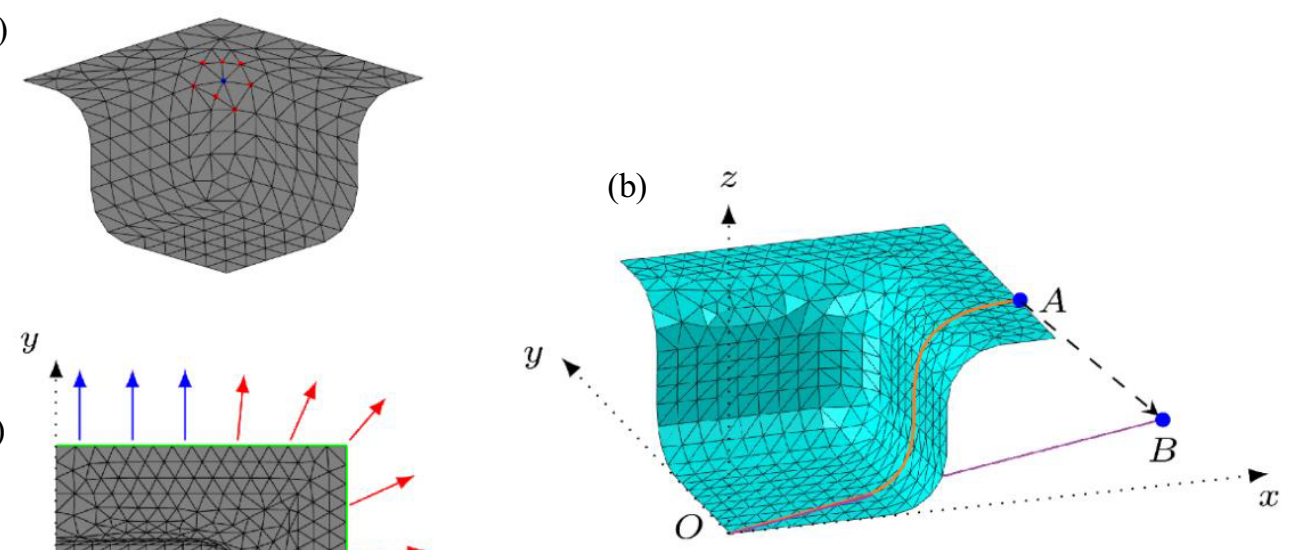

(c)

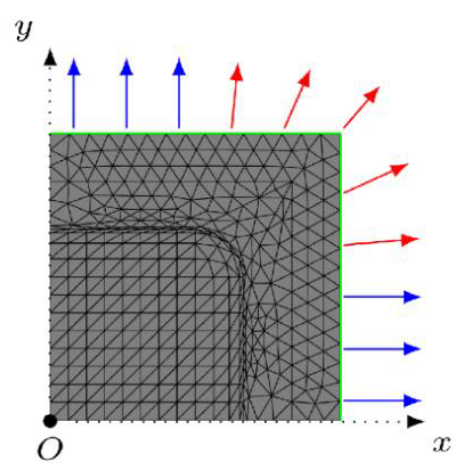

Figure 3. The strategies used for instigating un-forming, combined from [7]. 

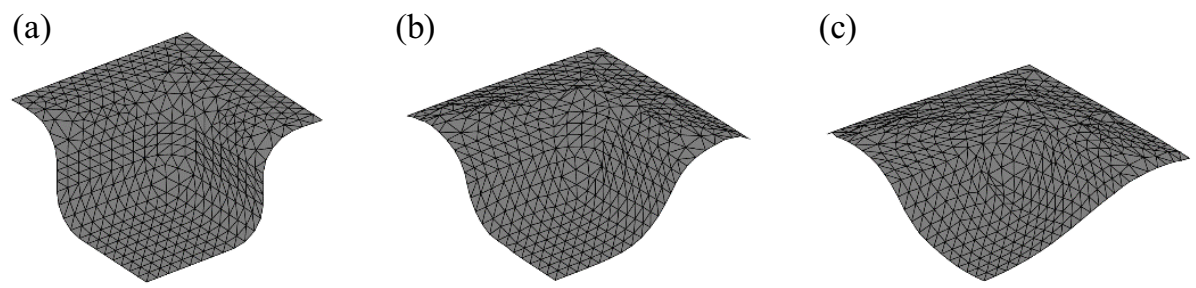

(d)

Figure 4. The deformation path for the "radial pull" strategy, modified from [7].

and improving the constraints on the process are listed below:

\subsection{Plastic strain}

There is little useful information in looking at the absolute values of strain, although empirically extreme values are troublesome. Instead, the standard technique for evaluating the material response and limitations is the Forming Limit Diagram (FLD). This examines the relative magnitudes of the major and minor strains for the metal. Much experimental work has been done to produce such plots for various materials. However, FLDs are usually based on the theoretical assumption that the strain path is linear or the corresponding experimental set up. According to Hosford and Caddel [17], changes in the strain path have a very large effect on the forming limits. Hence it is essential that the strain paths are also examined.

A plot of major and minor strains is used here to observe typical local deformation on the workpiece and evaluate the success of the optimization procedure. In Figure 5, the major and minor in-plane true plastic strains for each element and for all time steps $\left(\phi_{1}\right.$ and $\phi_{2}$ respectively) are shown. Histograms were added on each axes to show the relative frequency of each value. In addition, the mean of all the strains was plotted as a continuous black line, while a dashed red line corresponding to $\phi_{1}=-\phi_{2}$ is included.

The large number of data points, representing 780 elements at 65 time steps obfuscates any patterns, other than the fact that almost all the strain pairs are in the fourth quadrant, as expected for a "close to pure shear" deformation and close to the pure shear line. At the same time, it is apparent that some minor deviation from $\phi_{1}=-\phi_{2}$ does exist at some locations. However, the average strain values across the workpiece show no noticeable deviation the pure shear line at any time step.

A closer look at this metric can be obtained by plotting a smaller subset of element strain paths. Representative elements are selected from the different geometrical regions on the part. Specifically, points of positive, negative and zero Gaussian curvature are selected. In Figure 6 the locations of the five points are shown, along with the corresponding strain paths. Similar to Figure 5, linear strain paths are observed and the behaviour appears independent of location. There is a noticeable jump in magnitude between the different elements. In particular, elements 3 and 4, with zero Gaussian curvature in the final part geometry seem to experience greater deformation than elements 1 and 2 with positive and negative $\mathrm{GC}$ respectively.

For the same set of points, the through thickness deformation is also evaluated in Figure 7, where a plot of the through-thickness plastic strain is shown. All the selected points show some deviation from the constant thickness constraint, but this is typically smaller than $0.5 \%$. Most interestingly, elements 3 and 4 suffer the greatest through-thickness deformation - thickening and thinning respectively - but elements 1 and 2 are much closer to a constant thickness, throughout the process.

Figure 7 also allows a direct evaluation of the accuracy of the optimization algorithm. Given that the constraint tolerance was set at 0.001 , it is clear that the optimizations succeeds in achieving the constant area constraint. Any discrepancies for $\phi_{3}$, can perhaps be attributed to a cumulative increase in deviation from the constraint.

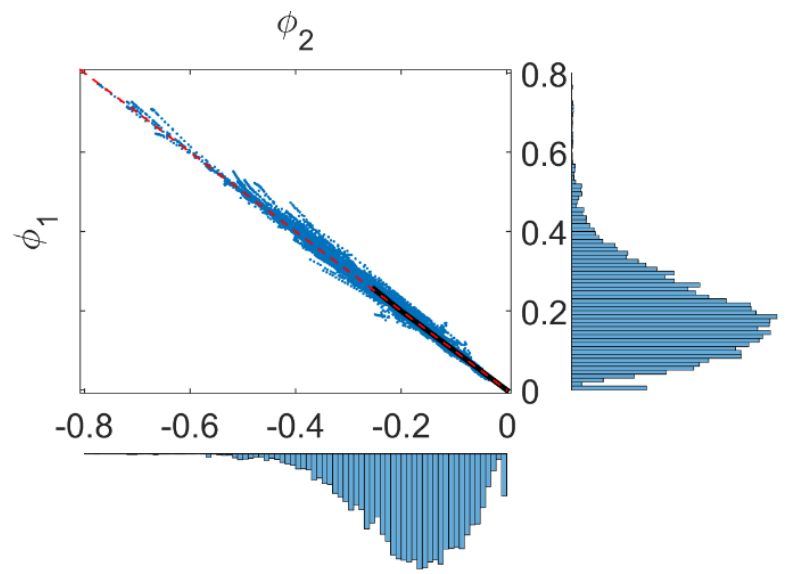

Figure 5. The major, $\phi_{1}$ and minor, $\phi_{2}$ plastic strains for all elements and for all time steps in the forming.

The plastic strain magnitudes and combinations observed here significantly exceed those observed in common forming operations, or those that can be sustained by the specified material. For heat-treated $\mathrm{Al}$ 1100 , principal strains at pure shear failure do not exceed 0.4 [20], indicating that the first iteration describes an unrealistic un-forming operation. At the same time however, from Figure 5, the locations that develop these extreme values are very few and could perhaps be eliminated completely with improved un-forming strategies. The average strains across the workpiece are well-within tolerated values. 

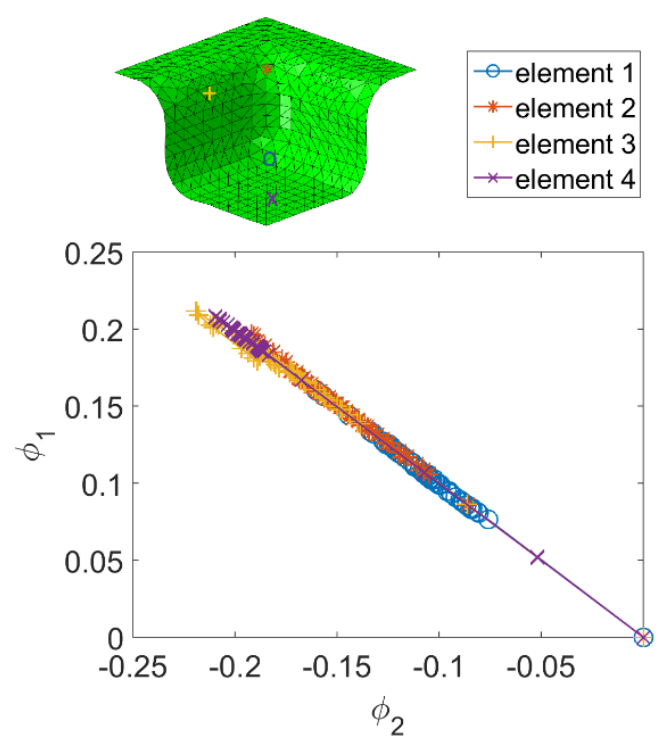

Figure 6. Strain paths of individual elements from representative geometrical locations.

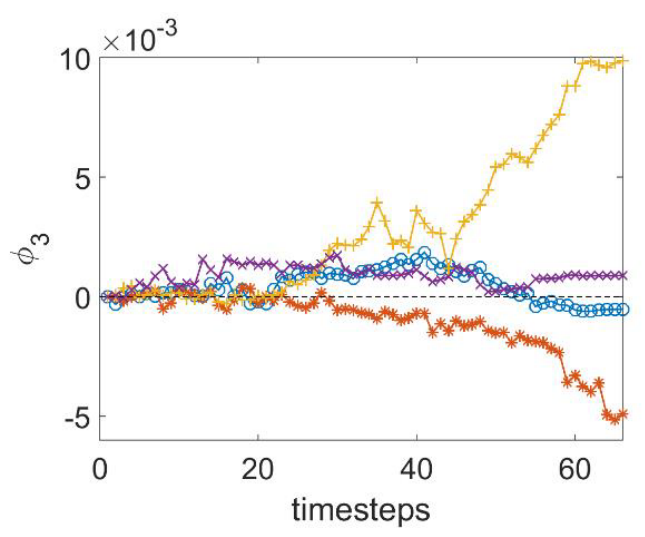

Figure 7. The through-thickness plastic strain for the same set of points as shown in Figure 6.

\subsection{Required external forces}

Beyond the material limits, for the process to be admissible, the required external forces need to be examined. In [7] a qualitative plot of the forces at one instant in the deformation where shown. A similar plot is shown in Figure 8(a).

The magnitudes of the forces have an apparent correlation with different geometrical features. For example, the highest force magnitudes are concentrated in the area of the brim, where the walls of the cup meet the flange. In contrast, and perhaps predictably, the bottom of the cup requires relatively low forces. This pattern seems to be consistent with knowledge from other forming processes, such as deep drawing.

A challenging result here however is that the direction of the forces with respect to the surface tends to alternate throughout the workpiece. Figure 8(c) displays a diagram of the nodal forces on a single element. Two of the nodal forces point to one side of the element, while the third points in the opposite side. This pattern is typical for the majority of the elements and perhaps a consequence of attempting to enforce pure shear everywhere. However, it makes the arrangement of conventional tooling impractical.

The directionality of the tangential component of the force is also an interesting result since there is no discernible pattern. In Figure 8(b) a plan view of the tangential forces during step 20 in the forming process confirms that even within specific regions, the range of directions needed for the suggested deformation does not point to any known tooling options. On the other hand, when the angle the tangential component of the force makes with the x-axes is plotted in Figure 9, an almost uniform distribution is observed.

The same Figure shows the corresponding quantitative distribution of magnitudes for the nodal forces - again for step 20. A sense of the magnitude of the force field throughout the forming process, can be obtained by looking at the average of the nodal forces. The median magnitude of nodal forces is $671 \mathrm{~N}$ at the first forming step and $609 \mathrm{~N}$ at the last step, and no great deviation beyond this range is observed at any other step.

Altan and Tekkaya [19] report punch forces for the deep drawing of a comparable part with similar material properties in the range of $50-200 \mathrm{kN}$. Thus for a comparable mesh coarseness, an average nodal force in the deep drawing operation is in the $117-467 \mathrm{~N}$ range. Hence, the forces suggested by the un-forming method are in proximity to those employed in existing processes, although their local application remains a challenge.
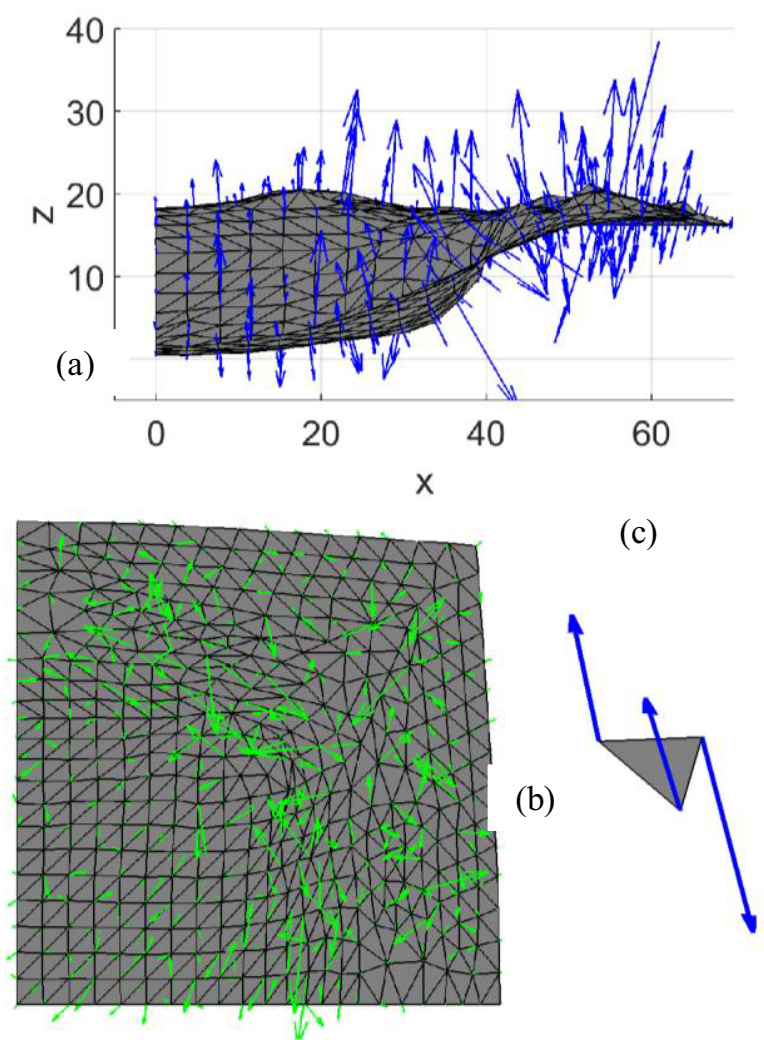

Figure 8. (a) Profile view of required nodal forces (step $20 / 65$ ) and (b) plan view of tangential forces. Typical direction of forces on a single element is shown in (c). 


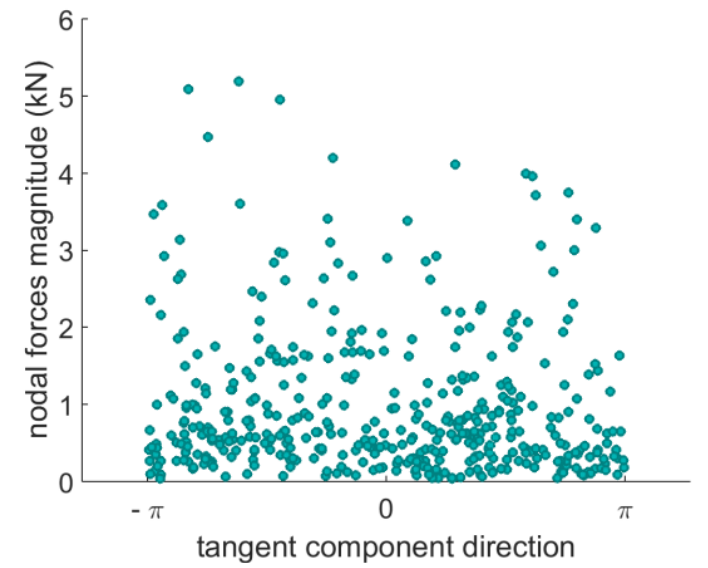

Figure 9. The magnitude of the nodal forces plotted against the tangent component's angle to the X-axes (step 20/65).

\subsection{Required friction coefficient assuming single tool at each node}

The process of coming up with practical tools necessitates an investigation of the friction required to achieve the external forces shown in the previous section. Such an investigation assumes that the forces will be provided at each node by a single tool. The tangential and normal components of nodal forces from the Abaqus simulation are first obtained and then divided to produce the required friction coefficient at each location on the surface.

Figure 10 is a "colormap" of the required friction coefficient at each node with the assumptions above. A large number of elements require infeasible values of friction. Thus, alternative tooling which allows the application of greater forces in the plane of the surface rather than out-of-plane need to be considered. In turn, this might mean the use of a holder (either locally or globally), as well as the necessity of fine local control of tool loads and directions.

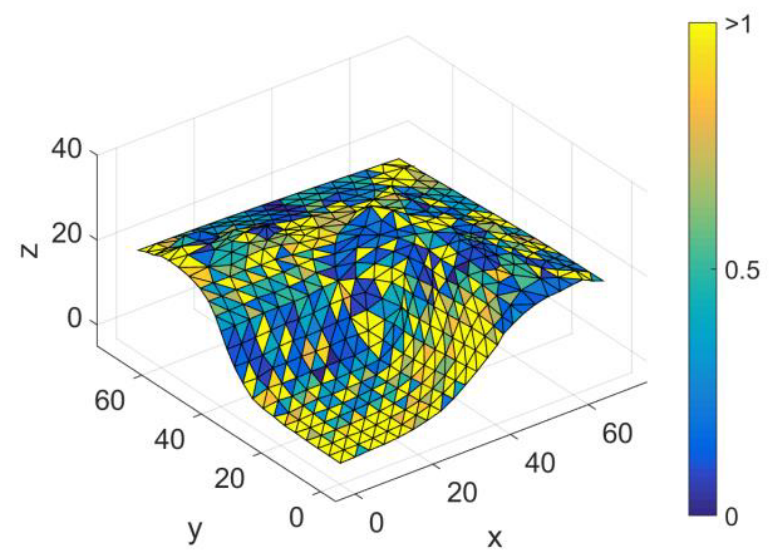

Figure 10. Colormap of required friction coefficients on surface of workpiece (time step 30 of 65)

\section{Discussion}

This paper extended work in [7], which proposed a new method for designing sheet forming processes by un- forming a desired part while minimizing the plastic work done and applying pre-selected kinematic constraints. Here, the results from [7] are evaluated with consideration for the practical implications.

This examination suggests that the current optimization method succeeds in satisfying the pure shear constraint across the workpiece. However, there is no direct way of measuring the proximity of the un-forming solution to the true minimum plastic work at each step.

Adherence to the constraints can potentially be improved further by a less naïve un-forming strategy. For example, instead of a hard constraint on the trajectory of peripheral nodes, their average distance from the centre might be gradually increased instead. But to guarantee the success of the optimization, a convex expression of the plastic work needs to be constructed and provided to a suitable solver that guarantees a global minimum. Similar efforts can be found in the literature [21].

For addressing the complexity of force directionality, an additional future constraint could prevent the deformation of certain regions, thus producing a more localized tool operation. Mathematically, this means allowing certain elements to only move without being distorted.

Finally, friction-based tools have been rejected as a practical approach to achieving such deformation. For pure shear deformation and the required in plane forces, gripping of the sheet might be necessary.

In summary, this paper analysed results from [7] and suggested possible steps in developing this method further and producing realistic processes in this automated manner.

\section{Acknowledgements}

This research was supported by the EPSRC grant EP/K018108/1.

\section{References}

1. R. L. Milford, J. M. Allwood, and J. M. Cullen, Resour. Conserv. Recycl., 55, 12 (2011)

2. J. A. Polyblank and J. M. Allwood, CIRP Ann. Manuf. Technol., 64, 1 (2015).

3. J. M. Allwood, G. P. F. King, and J. R. Duflou, Proc. Inst. Mech. Eng. Part B J. Eng. Manuf., 219, 2 (2005)

4. J. Jeswiet, M. Geiger, U. Engel, M. Kleiner, M. Schikorra, J. Duflou, R. Neugebauer, P. Bariani, and S. Bruschi, CIRP J. Manuf. Sci. Technol., 1, 1 (2008)

5. J. M. Allwood and H. Utsunomiya, Int. J. Mach. Tools Manuf., 46, 15 (2006).

6. P. Groche, D. Fritsche, E. a. Tekkaya, J. M. Allwood, G. Hirt, and R. Neugebauer, CIRP Ann. - Manuf. Technol., 56, 2 (2007).

7. E. G. Loukaides, J. M. Allwood, Int. J. Mech. Sc. (submitted, 2016)

8. J. McCartney, B. K. Hinds, and B. L. Seow, Comput. Des., 31, 4 (1999)

9. C. C. L. Wang, S. S. F. Smith, and M. M. F. Yuen, CAD Comput. Aided Des., 34, 11 (2002) 
10. B. K. Hinds, J. McCartney, and G. Woods, Comput. Des., 23, 8 (1991)

11. P. Azariadis and N. Aspragathos, Computer-Aided Design, 29, 10 (1997)

12. P. N. Azariadis, A. C. Nearchou, and N. A. Aspragathos, Comput. Ind., 47, 3 (2002)

13. C. H. Lee and H. Huh, J. Mater. Process. Technol., 82, 145-155 (1998)

14. Z.-Y. Cai, M.-Z. Li, and H.-M. Zhang, Finite Elem. Anal. Des., 43, 4 (2007)

15. K. Chung and O. Richmond, Int. J. Mech. Sci., 34, 7 (1992)

16. K. Chung and O. Richmond, Int. J. Mech. Sci., 34, 8 (1992)

17. W. Hosford and R. Caddell, Metal forming: mechanics and metallurgy (2011)

18. K. Lange, Handbook of metal forming (McGrawHill, 1985)

19. T. Altan and A. E. Tekkaya, Eds., Sheet Metal Forming: Fundamentals (ASM International, 2012)

20. A. I., Durán, J. W. Signorelli, D. J. Celentano, M. A. Cruchaga and M. François, J. Mat. Eng. Perf. 24, 10 (2015)

21. A. Raithatha and S. R. Duncan, Int. J. Numer. Methods Eng., 78, 955-979 (2009). 